[4] Hauben M. Comment. Papular rash and bilateral pleural effusion associated with clozapine. Ann Pharmacother 1999;33: 1374.

[5] Murko A, Clarke S, Black DW. Clozapine and pericarditis with pericardial effusion. Am J Psychiatry 2002;159:494.

[6] Stanislav SW, Gonzalez-Blanco M. Papular rash and bilateral pleural effusion associated with clozapine. Ann Pharmacother 1999;33:1008-9.

[7] Thompson J, Chengappa KN, Good CB, Baker RW, Kiewe RP, Bezner J, et al. Hepatitis, hyperglycemia, pleural effusion, eosinophilia, hematuria and proteinuria occurring early in clozapine treatment. Int Clin Psychopharmacol 1998;13:95-8.

[8] Wehmeier PM, Heiser P, Remschmidt H. Pancreahtis followed by pericardial effusion in an adolescent treated with clozapine. J Clin Psychopharmacol 2003;23(1):102-3.

Eric Boot

Lieuwe de Haan*

Yener Guzelcan

Willem F. Scholte

Hanneke Assies

Departamento de Psiquiatría, Centro Médico Académico, Universidad de Amsterdam, P. O. Box 22700, 1100 DE Amsterdam, Países Bajos

\section{El modafinilo como alternativa a la fototerapia para la depresión invernal}

Palabras clave: Modafinilo; Depresión; Trastorno afectivo estacional

El modafinilo es un nuevo psicoestimulante comercializado para la somnolencia diurna asociada con la narcolepsia. También ha mostrado eficacia en la depresión como tratamiento de aumento [3] o en monoterapia [1]. Considerando sus propiedades psicoestimulantes, parece sensato probar este compuesto en subtipos de depresión con fatiga acusada, hipersomnia o ambas cosas, como la depresión atípica o la depresión invernal.

Se vio ambulatoriamente al señor $X$, un hombre de 46 años, el 2 de enero en nuestro departamento de "trastorno del estado de ánimo" por depresión mayor según los criterios de la CIE-10, con intensidad moderada sin síndrome somático (F 33.10) a pesar de 6 semanas de tratamiento con $20 \mathrm{mg}$ /día de citalopram. El paciente satisfizo los criţerios provisionales de la CIE-10 para trastorno afectivo estacional. Aparecieron recurrencias depresivas cada año durante más de 20 años, con recuperación completa en primavera y verano. El paciente cumplía también los criterios de atipicidad de Liebowitz [2]. La gravedad se evaluó con la Guía de Entrevista Estructurada para la Escala de Evaluación de la Depresión de Hamilton - Versión de Trastornos Afectivos Estacionales [4], la Escala de Depresión de Hamilton (HDS) habitual de 21 elementos más ocho elementos que evalúan los síntomas atípicos (SA) y con la Escala de
Ansiedad y Depresión Hospitalaria (HAD) [5]. Las puntuaciones iniciales en la escala de Hamilton fueron 17 en la escala de depresión y 16 en la escala para síntomas atípicos. La puntuación en la subescala de depresión de la HAD fue 12. La estacionalidad se evaluó con la Escala Estacional Global (GSS) de la Escala de Evaluación del Patrón Estacional. La puntuación de la GSS del señor X fue 23.

Se añadió fototerapia de luz brillante al tratamiento en curso de citalopram. Después de 14 sesiones matutinas diarias de luz blanca de 10.000 lux durante $30 \mathrm{~min}$, las puntuaciones depresivas no habían cambiado ( $\mathrm{HS}=18$; $\mathrm{SA}=$ $19 ; \mathrm{HAD}=12$ ). La fototerapia se interrumpió entonces y se añadió modafinilo $(200 \mathrm{mg} / \mathrm{día})$ al citalopram actual. Dos semanas después ( 3 de febrero de 2003) el paciente mostraba remisión ( $\mathrm{HS}=3, \mathrm{SA}=3, \mathrm{HAD}=2$ ), pero sentía todavía algo de fatiga, lo que llevó a aumentar la dosis de modafinilo ( $300 \mathrm{mg} / \mathrm{día})$. Otras 5 semanas después, estaba bien todavía ( $\mathrm{HS}=4 ; \mathrm{SA}=0$ ) sin fatiga bajo el mismo régimen.

La mejoría espectacular se produjo después de 10 semanas de tratamiento con citalopram y más de 2 meses antes del periodo de remisión habitual del paciente. Por tanto, esta mejoría se puede atribuir primaria o incluso exclusivamente al modafinilo. Según este caso clínico, el modafinilo se puede considerar para la depresión atípica o invernal como un tratamiento de aumento o alternativo.

\section{Bibliografía}

[1] Kaufman KR, Menza MA, Fitzsimmons A. Modafinil monotherapy in depression. Eur Psychiatry 2002;17:167-9.

[2] Liebowitz MR, Quitkin FM, Stewart JW, McGrath PJ, Harrison WM, Markowitz JS, et al. Antidepressant specificity in atypical depression. Arch Gen Psychiatry 1988;45:129-37.

[3] Menza MA, Kaufman KR, Castellanos AM. Modafinil augmentation of antidepressant treatment in depression. J Clin Psychiatry 2000;61: 378-81.

[4] Williams JBW, Link MJ, Rosenthal NE, Amira L, Terman M. Structured interview guide for the Hamilton Depression Rating Scale, Seasonal Affective Disorders Version (SIGH-SAD). New York: New York State Psychiatric Institute; 1994.

[5] Zigmond AS, Snaith RR The hospital anxiety and depression scale. Acta Psychiatr Scand 1983;67:361-70.

C. Even

S. Friedman

Clínica de las Enfermedades Mentales y del Encéfalo, Servicio del Pr. J. D. Guelfi, Centro Hospitalario Santa Ana, 100 rue de la santé, 75674 Paris cedez 14, Francia

R. Dardennes

J. D. Guelfi

Universidad Paris V, U. F. R. Cochin, Port Royal

París, Francia 